\title{
Full Counting Statistics of a Superconducting Beam Splitter
}

\author{
J. Börlin, W. Belzig, and C. Bruder \\ Departement für Physik und Astronomie, Klingelbergstrasse 82, 4056 Basel, Switzerland
}

(Received 31 January 2002; published 30 April 2002)

\begin{abstract}
We study the statistics of charge transport in a mesoscopic three-terminal device with one superconducting terminal and two normal-metal terminals. We calculate the full distribution of transmitted charges into the two symmetrically biased normal terminals. In a wide parameter range, we find large positive cross correlations between the currents in the two normal arms. We also determine the third cumulant that provides additional information on the statistics not contained in the current noise.
\end{abstract}

DOI: $10.1103 /$ PhysRevLett.88.197001

The number of charges transferred in a transport process fluctuates due to quantum-mechanical uncertainty and statistics. Therefore, the outcome of a current measurement accumulated over some time period $t_{0}$ is in general described by a probability $P(N)$, where $N$ is the total number of charges transferred. $P(N)$ is called the full counting statistics (FCS) of the transport process [1]. The first two moments of the FCS are related to the average current and the current noise and are accessible to present experimental techniques. Higher-order correlations are likely to be measured in the future. Several schemes to measure either higher correlators or the full distribution have been proposed recently [1-6].

The current noise, i.e., the second moment of the FCS, is of particular interest. It can be used as a diagnostic tool to probe the nature and the quantum statistics of the charge carriers [7] and the existence of entanglement [8]. For superconductor $(S)$-normal metal $(N)$ heterostructures, a doubling of the shot noise in comparison to the normal case was predicted [9] and measured in diffusive heterostructures [10]. Recent calculations taking into account the proximity effect in such structures [11] are in good agreement with experimental results [12]. Multiterminal $S-N$ structures have been suggested to produce entangled electron pairs $[13,14]$.

So far, cross correlations, i.e., current correlations involving different terminals, were measured only in normal single-channel heterostructures [15]. These have confirmed the prediction [16] that current cross correlations in a fermionic system are always negative. To our knowledge, there is no measurement of cross correlations in a system with superconducting contacts up to now. Theoretically, positive cross correlation with a single-channel beam splitter for Andreev pairs injected from a superconductor have been predicted [17]. In a setup in which cross correlations between a normal lead and a tunneling probe are considered, the sign of the correlations was found to depend crucially on the sample geometry [18]. A numerical study found positive cross correlations in a threeterminal device with a few channels with ferromagnetic contacts [19].
PACS numbers: 74.50. $+\mathrm{r}, 05.40 .-\mathrm{a}, 72.70 .+\mathrm{m}, 73.23 .-\mathrm{b}$

In this Letter we find the full counting statistics of a many-channel beam splitter that divides a supercurrent in two normal quasiparticle currents. We calculate the distribution of the transmitted charges taking the proximity effect into account. For comparison we also calculate the FCS for the case in which the superconducting terminal is replaced by a normal one.

The setup of our three-terminal device with one superconducting and two normal-metal terminals is shown in Fig. 1. All three terminals are connected by tunnel junctions to a small normal-metal island. We assume the island to be large enough that we can neglect charging effects and small enough that we can treat the Green's functions of the island as constant. Thus, we are restricted to energies below the Thouless energy of the island. The system is then appropriately described by the circuit theory of mesoscopic transport [20], within which the counting statistics is easily accessible $[2,11,21]$.

The circuit elements that are important for our case are normal (superconducting) terminals and tunnel connectors. The terminals are described by quasiclassical Green's functions, which depend on the type of the terminal $(N$ or $S$ ), chemical potential, temperature $T$, and a counting field $\chi$. We assume zero temperature and a symmetric

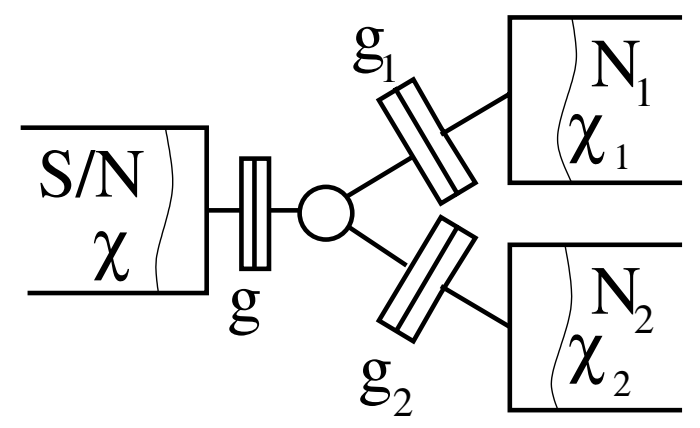

FIG. 1. Three-terminal beam splitter. One superconducting or normal terminal $(S$ or $N)$ and two normal terminals $\left(N_{1}\right.$ and $\left.N_{2}\right)$ are tunnel coupled by conductances $g, g_{1}$, and $g_{2}$ to a common central node. A current is passed from $S / N$ into the two normal leads that are kept at the same voltage. Ideal passive charge counters are indicated by the counting fields $\chi, \chi_{1}$, and $\chi_{2}$. 
bias at the two normal terminals. Since we are interested in correlations between currents in different terminals, we introduce different counting fields. The voltage is chosen such that $e V \ll \Delta$. Charge transport (at $T=0$ ) occurs then only in the interval $|E| \leq e V$ and we need to consider only this energy interval below.

The Green's functions of the two normal terminals are then given by

$$
\check{G}_{1,2}=e^{i \chi_{1,2} \check{\check{T}}_{K} / 2} \check{G}_{N} e^{-i \chi_{1,2} \check{\tau}_{K} / 2},
$$

where $\breve{G}_{N}$ is the same for both normal terminals. At zero temperature $\breve{G}_{N}=\hat{\sigma}_{3} \bar{\tau}_{3}+\left(\bar{\tau}_{1}+i \bar{\tau}_{2}\right) \hat{\mathbb{1}}$ for $|E| \leq e V$ and $\breve{G}_{N}=\hat{\sigma}_{3} \bar{\tau}_{3}+\operatorname{sgn}(E) \hat{\sigma}_{3}\left(\bar{\tau}_{1}+i \bar{\tau}_{2}\right)$ for $|E|>e V$. Here $\hat{\sigma}_{i}\left(\bar{\tau}_{i}\right)$ denote Pauli matrices in Nambu (Keldysh) space. The counting rotation matrix is $\check{\tau}_{K}=\hat{\sigma}_{3} \bar{\tau}_{1}$. The superconducting terminal in equilibrium is characterized by $\breve{G}_{S}=\hat{\sigma}_{1} \overline{\mathbb{1}}$ and a counting field $\chi$ that enters as in (1).

If one node is connected to $M$ terminals by means of tunnel connectors, one can find a general form of the FCS, i.e., the probability $P\left(N_{1}, \ldots, N_{M}\right)$ that $N_{1(2, \ldots, M)}$ charges are counted in terminal $1(2, \ldots, M)$. The unknown Green's function of the central node is denoted by $\check{G}_{c}$. The matrix currents into the central node are given by $\breve{I}_{k}=\frac{g_{k}}{2}\left[\breve{G}_{c}, \breve{G}_{k}\right]$, where the index $k=1, \ldots, M$ labels the terminals and $g_{k}$ is the conductance of the respective junction. The Green's function of the central node is determined by matrix current conservation on the central node, reading $\sum_{k=1}^{M} \breve{I}_{k}=\frac{1}{2}\left[\sum_{k=1}^{M} g_{k} \breve{G}_{k}, \breve{G}_{c}\right]=0$. Employing the normalization condition $\breve{G}_{c}^{2}=1$, the solution is

$$
\check{G}_{c}=\frac{\sum_{k=1}^{M} g_{k} \check{G}_{k}}{\sqrt{\sum_{k, m=1}^{M} g_{k} g_{m}\left\{\check{G}_{k}, \check{G}_{m}\right\} / 2}} .
$$

To find the cumulant-generating function (CGF) $S$ of $P\left(N_{1}, \ldots, N_{M}\right)$ we integrate the equations $\left(-i t_{0} / e\right) \times$ $\partial S\left(\chi_{1}, \ldots, \chi_{M}\right) / \partial \chi_{k}=\int d E \operatorname{Tr} \check{\tau}_{K} \check{I}_{k} / 8 e$ [22]. We obtain

$$
\begin{aligned}
S\left(\chi_{1}, \ldots, \chi_{M}\right)= & -\frac{t_{0}}{e} \int \frac{d E}{2} \\
& \times \operatorname{Tr} \sqrt{\sum_{k, m=1}^{M} \frac{g_{k} g_{m}}{2}\left\{\check{G}_{k}, \check{G}_{m}\right\}} .
\end{aligned}
$$

This is the general result for an $M$-terminal geometry in which all terminals are tunnel coupled to a common node.

We now evaluate Eq. (3) for our three terminal setup. Introducing $p_{i}=2 g g_{i} /\left[g^{2}+\left(g_{1}+g_{2}\right)^{2}\right]$ we find

$$
S\left(\chi_{1}, \chi_{2}, \chi\right)=-\frac{V t_{0} \sqrt{g^{2}+\left(g_{1}+g_{2}\right)^{2}}}{\sqrt{2 e}} \sqrt{1+\sqrt{1+\left(p_{1} e^{i\left(\chi_{1}-\chi\right)}+p_{2} e^{i\left(\chi_{2}-\chi\right)}\right)^{2}-\left(p_{1}+p_{2}\right)^{2}}}
$$

This result for the cumulant-generating function incorporates all statistical transport properties for our present setup. The inner argument contains counting factors for the different possible processes. A term $\exp \left[i\left(\chi_{k}+\chi_{l}-2 \chi\right)-1\right]$ corresponds to an event in which two charges leave the superconducting terminal and one charge is counted in terminal $k$ and one charge in terminal $l$. The prefactors are related to the corresponding probabilities. For instance, $p_{1}$ is proportional to the probability of a coherent tunneling event of an electron from the superconductor into terminal 1. A coherent pair-tunneling process is therefore weighted with $p_{1}^{2}$. This is accompanied by counting factors which describe either the tunneling of two electrons into terminal 1(2) [counting factor $\left.\exp \left(i 2\left(\chi_{1(2)}-\chi\right)\right)-1\right]$ or tunneling into different terminals [counting factor $\exp \left(i\left(\chi_{1}+\chi_{2}-2 \chi\right)\right)-1$ ]. The double square-root function shows that these different processes are nonseparable.

It is interesting to compare Eq. (4) with the case in which the superconductor is replaced by a normal metal. The resulting CGF is

$$
S^{N}\left(\chi_{1}, \chi_{2}, \chi\right)=-\frac{V t_{0}}{2 e}\left(g+g_{1}+g_{2}\right) \times \sqrt{1+p_{1}^{N}\left(e^{i\left(\chi_{1}-\chi\right)}-1\right)+p_{2}^{N}\left(e^{i\left(\chi_{2}-\chi\right)}-1\right)},
$$

where $p_{1(2)}^{N}=4 g g_{1(2)} /\left(g+g_{1}+g_{2}\right)^{2}$. Thus, one of the square roots in Eq. (4) can be attributed to the multiple tunnel-junction geometry, which is already present in the normal configuration. The second square root in the CGF for the superconducting case must then be due to the proximity effect.

We now evaluate some average transport properties of the $S \mid N N$ system and compare them to the $N \mid N N$ case. The currents into the different terminals are obtained from derivatives of the CGF: $I_{k}=$ $\left(-i e / t_{0}\right) \partial S /\left.\partial \chi_{k}\right|_{\chi_{1}=\chi_{2}=\chi=0}$. The transconductances $G_{k}=I_{k} / V$ into terminal $k(=1,2)$ are then given by

$$
G_{k}^{S}=\frac{g^{2} g_{k}\left(g_{1}+g_{2}\right)}{\left[g^{2}+\left(g_{1}+g_{2}\right)^{2}\right]^{3 / 2}}, \quad G_{k}^{N}=\frac{g g_{k}}{g+g_{1}+g_{2}} .
$$

The superscript $S(N)$ denotes the $S \mid N N(N \mid N N)$ case. Noise and cross correlations are obtained from second derivatives of the CGF, i.e., $P_{k l}^{I}=$ $\left(2 e^{2} / t_{0}\right) \partial^{2} S\left(\chi_{1}, \chi_{2}, \chi\right) /\left.\partial \chi_{k} \partial \chi_{l}\right|_{\chi_{1}=\chi_{2}=\chi=0}$. We define Fano factors $F_{k l}=P_{k l}^{I} / 2 e I$, and we denote the Fano factor of the total current with $F=F_{11}+F_{22}+2 F_{12}$. We also calculate the third cumulant of the total charge transfer (normalized to the Poisson value) 
$C_{3}=\left(i e / I t_{0}\right) \partial^{3} S(0,0, \chi) /\left.\partial \chi^{3}\right|_{\chi=0}$. The results in the superconducting case are

$$
\begin{aligned}
& F_{12}^{S}=\frac{g_{1} g_{2}}{\left(g_{1}+g_{2}\right)^{2}}\left(1-5 x^{2}\right), \quad F^{S}=2-5 x^{2}, \\
& C_{3}^{S}=4-30 x^{2}+63 x^{4}, \quad x=\frac{g\left(g_{1}+g_{2}\right)}{g^{2}+\left(g_{1}+g_{2}\right)^{2}} .
\end{aligned}
$$

In the $N \mid N N$ case, on the other hand, we find

$$
\begin{array}{ll}
F_{12}^{N}=-\frac{g_{1} g_{2}}{\left(g_{1}+g_{2}\right)^{2}} x_{N}, & F^{N}=1-2 x_{N}, \\
C_{3}^{N}=1-6 x_{N}+3 x_{N}^{2}, & x_{N}=\frac{g\left(g_{1}+g_{2}\right)}{\left(g+g_{1}+g_{2}\right)^{2}} .
\end{array}
$$

All other Fano factors can be deduced from $F_{12}$ and $F$ using the relations $\sum_{k} F_{k l}=0$ and $F_{k l}=F_{l k}$. The transport properties are summarized in Fig. 2 . In the figure the cross correlations are plotted as $f_{12}=F_{12}\left(g_{1}+\right.$ $\left.g_{2}\right)^{2} / g_{1} g_{2}$. Most remarkably, the cross correlations $F_{12}^{S}$ are positive if $x$ is small, whereas $F_{12}^{N}$ is always negative in the normal state. Here the Fano factor $F^{S}\left(F^{N}\right)$ is close to 2(1). Going to the regime $g \approx\left(g_{1}+g_{2}\right)$ suppresses the Fano factor $F^{S(N)}$ below 2(1) and leads to negative cross correlations $F_{12}^{S}$. In the limiting case $g=\left(g_{1}+g_{2}\right)$ the Fano factors are $F^{S}=3 / 4$ and $F^{N}=1 / 2$, and the cross correlations are $F_{12}^{S}=F_{12}^{N}=-g_{1} g_{2} / 4\left(g_{1}+g_{2}\right)^{2}$. The third cumulant is always positive, but shows a strong suppression around the resonant conductance ratio $g=$ $\left(g_{1}+g_{2}\right)$. In the limit of small $x\left(x_{N}\right)$ the third cumulant is 4 (1), corresponding to the effective charge squared transferred in a tunneling process [3]. However, the variation with $g /\left(g_{1}+g_{2}\right)$ in the $S \mid N N$ case is more pronounced than in the $N \mid N N$ case.

As an interesting side remark we point out that $F_{12}^{S}=0$ and $F^{S}=1$ for $x^{2}=1 / 5$. This looks like a signature of uncorrelated charge transfer in units of $e$. However, the third cumulant $C_{3}^{S}=13 / 25$ differs from the corresponding value for uncorrelated $1 e$-charge transfer, viz., $C_{3}=1$. Thus, higher correlations show that the charge transfer is still correlated.

We briefly discuss the influence of an asymmetry $g_{1} \neq$ $g_{2}$ of the beam splitter. The cross correlations are reduced, both in the $S \mid N N$ and in the $N \mid N N$ case. However, the positive cross correlations in the superconducting state persist for all values of the asymmetry. Cumulants of the total charge transfer like the conductance, $F^{S, N}$ and $C_{3}^{S, N}$ are independent of this asymmetry.

Using the CGF from Eq. (4), we can identify the physical processes leading to our previous results. We have seen from (7) that positive cross correlations are found if $g /\left(g_{1}+g_{2}\right)$ is not close to 1 . Then, $p_{1,2} \ll 1$ and we can expand Eq. (4) in $p_{1,2}$. Dropping the trivial dependence on $\chi$, the CGF can be written as
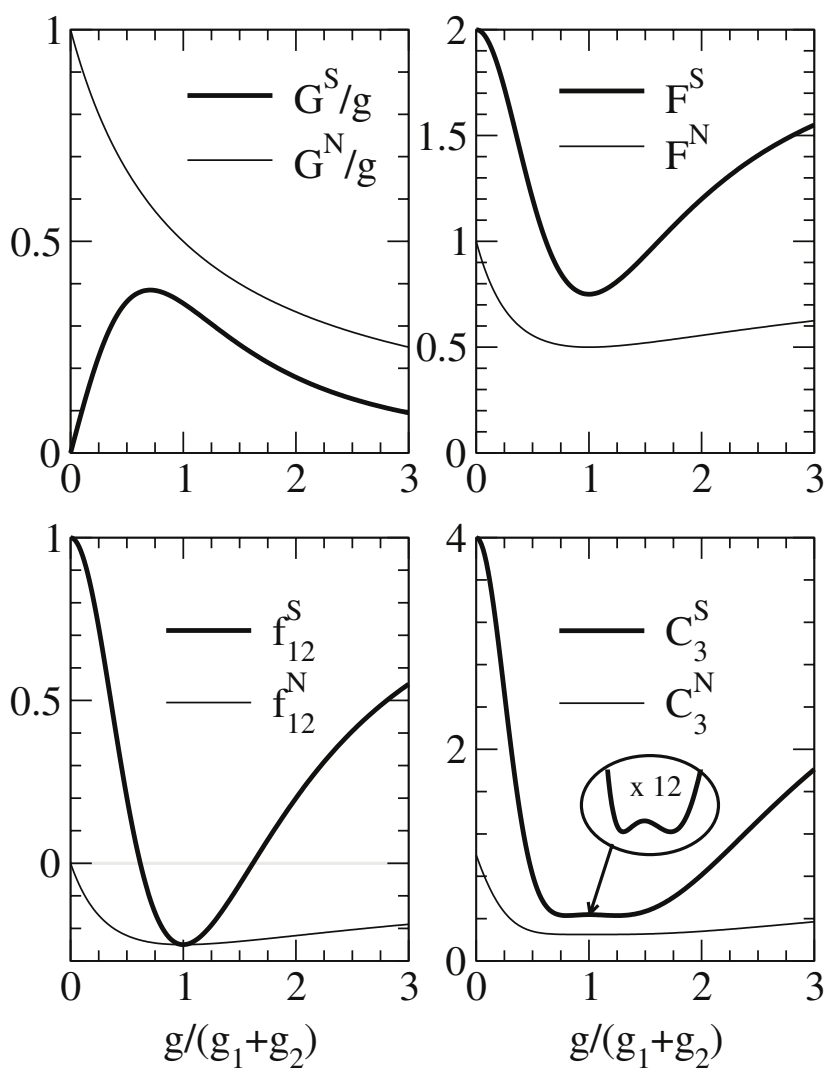

FIG. 2. Conductance, Fano factors, cross correlations, and third cumulant of the beam splitter. The thick lines correspond to the $S \mid N N$ case and the thin lines to the $N \mid N N$ case. The conductance (upper-left panel) in the superconducting case shows a maximum around $g=g_{1}+g_{2}$. In the normal state, the conductance varies between $g$ and $g_{1}+g_{2}$. In both cases, the current noise (upper-right panel) shows a suppression around $g=g_{1}+g_{2}$ as compared to the limiting values of 2 in the $S \mid N N$ case and 1 in the $N \mid N N$ case. Large positive cross correlations occur in the superconducting case (lower-left panel), whereas they are always negative in the normal case. Around $g=g_{1}+g_{2}$, the superconducting cross correlations become negative. Note that what is plotted here is $f_{12}^{S / N}=F_{12}^{S / N}\left(g_{1}+g_{2}\right)^{2} / g_{1} g_{2}$. The third cumulants (lower-right panel) are always positive. Around $g=g_{1}+g_{2}$ they are strongly suppressed. In the $S \mid N N$ case, $C_{3}^{S}$ has a double minimum here, as shown in the blowup.

$$
\begin{aligned}
S\left(\chi_{1}, \chi_{2}\right)= & -\frac{t_{0} V}{e} \frac{g^{2}}{\left[g^{2}+\left(g_{1}+g_{2}\right)^{2}\right]^{3 / 2}} \\
& \times\left(g_{1}^{2} e^{i 2 \chi_{1}}+g_{2}^{2} e^{i 2 \chi_{2}}+2 g_{1} g_{2} e^{i\left(\chi_{1}+\chi_{2}\right)}\right) .
\end{aligned}
$$

The CGF is composed of three different terms, corresponding to a charge transfer of $2 e$ either into terminal 1 or terminal 2 (the first two terms in the bracket) or separate charge transfer into terminals 1 and 2. According to the general principles of statistics, sums of CGFs of independent statistical processes are additive. Therefore, the CGF (9) is a sum of CGFs of independent Poissonian processes. Cross correlations are obtained from derivatives with respect to $\chi_{1}$ and $\chi_{2}$. Thus, the first two terms in (9) corresponding 
to two-particle tunneling either into terminal 1 or 2 do not contribute. It is only the last term which yields cross correlations, and those are positive. Poissonian statistics are the statistics of uncorrelated events, which in our case means all tunneling events are independent. Thus, a two-particle tunneling event into one of the normal terminals is not correlated with other tunneling events and does not contribute to cross correlations, but only to the autocorrelations. The two-particle tunneling into different terminals, however, is automatically positively cross correlated. The cross correlations are therefore positive.

The total probability distribution $P\left(N_{1}, N_{2}\right)$ corresponding to (9) can be found. It vanishes for odd values of $\left(N_{1}+N_{2}\right)$ and for even values it is

$$
P\left(N_{1}, N_{2}\right)=\frac{e^{-\bar{N} / 2}\left(\frac{\bar{N}}{2}\right)^{\left(N_{1}+N_{2}\right) / 2}}{\left(\frac{N_{1}+N_{2}}{2}\right) !}\left(\begin{array}{c}
N_{1}+N_{2} \\
N_{1}
\end{array}\right) T_{1}^{N_{1}} T_{2}^{N_{2}} .
$$

Here we have defined the average number of transferred electrons $\bar{N}=t_{0} G^{S} V / e$ and the probabilities $T_{1(2)}=$ $g_{1(2)} /\left(g_{1}+g_{2}\right)$ that one electron leaves the island into terminal 1(2). If one would not distinguish electrons in terminals 1 and 2 , the charge counting distribution can be obtained from (9) by setting $\chi_{1}=\chi_{2}=\chi$ and performing the integration. This leads to $P_{\text {tot }}^{S}(N)=$ $\exp (-\bar{N} / 2)(\bar{N} / 2)^{N / 2} /(N / 2)$ !, which corresponds to a Poisson distribution of an uncorrelated transfer of electron pairs. The full distribution (10) is given by $P_{\text {tot }}^{S}\left(N_{1}+N_{2}\right)$, multiplied with a partitioning factor, which corresponds to the number of ways $N_{1}+N_{2}$ identical electrons can be distributed among the terminals 1 and 2 , with respective probabilities $T_{1}$ and $T_{2}$. Note that $T_{1}+T_{2}=1$, since the electrons have no other possibility to leave the island.

In contrast to that, we obtain in the normal case for $t_{1,2}^{N} \ll 1$ the probability distribution

$$
P^{N}\left(N_{1}, N_{2}\right)=e^{-\bar{N}_{1}} \frac{\bar{N}_{1}^{N_{1}}}{N_{1} !} e^{-\bar{N}_{2}} \frac{\bar{N}_{2}^{N_{2}}}{N_{2} !} .
$$

Here we have abbreviated the average number transferred into terminal $i$ by $\bar{N}_{i}$. Thus, the distribution in the normal case is the product of two Poisson distributions of charge transfers into the two terminals. In the superconducting case such a factorization is not possible.

In conclusion, we have studied the full counting statistics of a three-terminal device with one superconducting and two normal leads. The system is biased such that a supercurrent is passed from the superconductor into the two normal leads, with no net current between the normal leads. Thus, the device acts as a sort of beam splitter. We have calculated the full distribution of transmitted charges using the extended Keldysh-Green's function method fully accounting for the proximity effect. Our main finding is large positive cross correlations of the currents in the two normal terminals in a wide parameter range. These should be easily accessible experimentally. These positive correlations originate from independent Poisson processes of coherent tunneling of charges into the different terminals.
These dominate the cross correlations, since two-particle tunneling into the same lead does not contribute to the cross correlations. We have also calculated the third cumulant which provides additional information on the current statistics not contained in the current noise.

We thank G. Burkard, D. Loss, and Yu. V. Nazarov for discussions. During the preparation of this manuscript, we became aware of similar work by P. Samuelsson and M. Büttiker [23]. Our work was supported by the Swiss NSF and the NCCR Nanoscience.

[1] L. S. Levitov, H. W. Lee, and G. B. Lesovik, J. Math. Phys. (N.Y.) 37, 4845 (1996).

[2] W. Belzig and Yu. V. Nazarov, Phys. Rev. Lett. 87, 197006 (2001).

[3] L.S. Levitov and M. Reznikov, cond-mat/0111057 (unpublished).

[4] Yu. V. Nazarov and M. Kindermann, cond-mat/0107133 (unpublished).

[5] C. W. J. Beenakker and H. Schomerus, Phys. Rev. Lett. 86, 700 (2001).

[6] A. Andreev and A. Kamenev, Phys. Rev. Lett. 85, 1294 (2000); Yu. Makhlin and A.D. Mirlin, ibid. 87, 276803 (2001); L. S. Levitov, cond-mat/0103617 (unpublished).

[7] Ya. M. Blanter and M. Büttiker, Phys. Rep. 336, 1 (2000).

[8] D. Loss and E. V. Sukhorukov, Phys. Rev. Lett. 84, 1035 (2000); G. Burkard, D. Loss, and E. V. Sukhorukov, Phys. Rev. B 61, R16303 (2000).

[9] V. A. Khlus, Sov. Phys. JETP 66, 1243 (1987); B. A. Muzykantskii and D.E. Khmelnitskii, Phys. Rev. B 50, 3982 (1994); M. J. M. de Jong and C. W. J. Beenakker, ibid. 49, 16070 (1994); K. E. Nagaev and M. Büttiker, ibid. 63, 081301(R) (2001).

[10] X. Jehl et al., Nature (London) 405, 50 (2000).

[11] W. Belzig and Yu. V. Nazarov, Phys. Rev. Lett. 87, 067006 (2001).

[12] A. A. Kozhevnikov, R. J. Schoelkopf, and D. E. Prober, Phys. Rev. Lett. 84, 3398 (2000).

[13] P. Recher, E. V. Sukhorukov, and D. Loss, Phys. Rev. B 63, 165314 (2001).

[14] G. B. Lesovik, T. Martin, and G. Blatter, Eur. Phys. J. B 24, 287 (2001).

[15] M. Henny et al., Science 284, 296 (1999); W. D. Oliver et al., Science 284, 299 (1999).

[16] M. Büttiker, Phys. Rev. Lett. 65, 2901 (1990); Phys. Rev. B 46, 12485 (1992).

[17] T. Martin, Phys. Lett. A 220, 137 (1996); M. P. Anantram and S. Datta, Phys. Rev. B 53, 16390 (1996); J. Torres and T. Martin, Eur. Phys. J. B 12, 319 (1999).

[18] T. Gramespacher and M. Büttiker, Phys. Rev. B 61, 8125 (2000).

[19] F. Taddei and R. Fazio, cond-mat/0111564 (unpublished).

[20] Yu. V. Nazarov, Phys. Rev. Lett. 73, 134 (1994); Yu. V. Nazarov, Superlattices Microstruct. 25, 1221 (1999).

[21] Yu. V. Nazarov, Ann. Phys. (Leipzig) 8, SI-193 (1999).

[22] Yu. V. Nazarov and D. Bagrets, cond-mat/0112223 (unpublished).

[23] P. Samuelsson and M. Büttiker, cond-mat/0203188 (unpublished). 